\title{
Stability of flat-band edge states in topological superconductors without inversion center
}

\author{
Raquel Queiroz ${ }^{1, \text { * }}$ and Andreas P. Schnyder ${ }^{1}$, \\ ${ }^{1}$ Max-Planck-Institut für Festkörperforschung, Heisenbergstrasse 1, D-70569 Stuttgart, Germany
}

(Dated: August 23, 2021)

\begin{abstract}
Nodal superconductors without inversion symmetry exhibit nontrivial topological properties, manifested by topologically protected flat-band edge states. Here we study the effects of edge roughness and strong edge disorder on the flat-band states using large-scale numerical simulations. We show that the bulk-edge correspondence remains valid for rough edges and demonstrate that midgap states generically appear at the boundary of nodal noncentrosymmetric superconductors, for almost all edge orientations. Moderately strong nonmagnetic disorder shifts some of the edge states away from zero energy, but does not change their total number. Strong spin-independent edge disorder, on the other hand, leads to the appearance of new weakly disordered midgap states in the layers adjacent to the disordered edge, i.e., at the interface between the bulk topological superconductor and the one-dimensional Anderson insulator formed by the strongly disordered edge layers. Furthermore, we show that magnetic impurities, which lift the time-reversal symmetry protection of the flat-band states, lead to a rapid decrease of the number of edge states with increasing disorder strength.
\end{abstract}

PACS numbers: 03.65.vf,74.50.+r, 73.20.Fz, 73.20.-r:

\section{INTRODUCTION}

Topological superconductors have recently attracted considerable theoretical ${ }^{1 / \sqrt[8]{8}}$ and experimental ${ }^{9 ! 13}$ interest, due to the possibility of realizing exotic zero-energy edge states in these systems. Depending on the superconducting pairing symmetry, these edge states are either chiral or helical Majorana modes, or, in the case of nodal superconductors, form zero-energy flat bands. ${ }^{[14 \mid 23}$ One particularly interesting class of topological superconductors are noncentrosymmetric superconductors with strong spin-orbit coupling. $24 \mid 25$ Many of these compounds, e.g., $\mathrm{CePt}_{3} \mathrm{Si}^{26}{ }^{28} \mathrm{CeIrSi}_{3},{ }^{29[30}$ and $\mathrm{Li}_{2} \mathrm{Pt}_{3} \mathrm{~B}, 31,34$ are reported to have unconventional pairing symmetries with sizable spin-triplet pairing components and line nodes in the superconducting gap. As a result of Rashbatype spin-orbit interactions, the flat-band edge states in these systems are spin-nondegenerate and exhibit a helical spin polarization, where the spin orientation varies as a function of edge momentum. ${ }^{35 \sqrt[3]{38}}$ As a consequence of the nontrivial spin texture, the lowest-order matrix element for spin-independent backscattering among the surface states is suppressed $\mathbf{3 7 . 3 8}$

The boundary states of clean topological superconductors in a ribbon or slab geometry are well studied theoretically. For example, for a two-dimensional $\left(d_{x y}+p\right)$-wave superconductor on the square lattice, it was shown that flat-band edge states appear at the (10) and (01) edges, but are absent at the (11) edge (see Fig. 1). This can be understood in terms of a bulkedge correspondence: The topological properties of the quasiparticle wave functions in the bulk, which are characterized by a one-dimensional winding number, directly imply the existence of zero-energy states at the edge [14/20122123]39 $_{\text {However, }}$ in the presence of edge disorder or for a superconducting dot with a closed boundary, it is not clear whether the bulk-edge correspondence still applies. Strictly speaking, the topological winding number is ill-defined in the absence of translational symmetry, since it is given in terms of a momentum-space integral ${ }^{40}$ Nevertheless, sufficiently large disks of topological superconductors are expected to show the same edge properties as topological superconductors in an infinite ribbon ge- ometry. The study of edge roughness or edge disorder has direct relevance for experiments, since surfaces of unconventional superconductors are often either intrinsically disordered or can be intentionally disordered via the deposition of impurity atoms.

In this paper, using the $\left(d_{x y}+p\right)$-wave superconductor as a prototypical example, we study the edge properties of disordered nodal topological superconductors with rough irregular boundaries consisting of both (10) edge and (11) edge parts. By means of large-scale numerical simulations of a two-dimensional Bogoliubov-de Gennes (BdG) lattice model, we demonstrate the validity of the bulk-edge correspondence for rough edges, and show that the number of edge states is proportional to the length of the boundary (see Fig. 2). Secondly, we investigate the effects of strong nonmagnetic edge impurities, which influence both the edge and the bulk quasiparticle wave functions, leading to a nontrivial coupling between the two. We find that strong edge disorder localizes the states in the edge layer, but leads to the appearance of new weakly disordered ingap states in the second and third inward layers, just below the strongly disordered edge. That is, zeroenergy states appear at the interface between the bulk topological superconductor and the Anderson insulating state of the first layer (see Fig. 3). Weak nonmagnetic impurities, on the other hand, only spread the zero-energy edge states over an energy band of small finite width, leaving the total number of edge states unchanged. Finally, we also consider magnetic impurities, which lift the symmetry protection of the flat-band edge states. Due to spin-flip scattering, a finite density of impurity spins gives rise to a rapid decrease of the number of edge states with increasing disorder strength (see Fig. 47.

The outline of the paper is as follows. In Sec. II we start by introducing the $\mathrm{BdG}$ Hamiltonian of the $\left(d_{x y}+p\right)$-wave superconductor on the square lattice. In Sec. III] an analytical expression for the flat-band edge-state wave functions is given and the lowest-order matrix element for scattering among the flat-band edge states is determined. Using large-scale exact diagonalization, we investigate in Sec. IV the edge properties of superconducting dots with closed irregular boundaries. 
The effects of strong magnetic and nonmagnetic edge disorder are studied numerically in Sec. $\mathrm{V}$ by means of the recursive Green's function technique. We conclude with a brief discussion in Sec. VI. Some of the technical details are relegated to two Appendices.

\section{MODEL DEFINITION}

We study the stability of flat-band edge states in noncentrosymmetric superconductors by considering, as a representative example, the $\left(d_{x y}+p\right)$-wave superconductor on the square lattice with both spin-singlet and spin-triplet pairing components. ${ }^{15}$ In momentum space this topological superconductor is described by a $4 \times 4 \mathrm{BdG}$ Hamiltonian $\mathcal{H}=$ $\frac{1}{2} \sum_{\mathbf{k}} \Phi_{\mathbf{k}}^{\dagger} H_{\mathbf{k}} \Phi_{\mathbf{k}}$, with

$$
H_{\mathbf{k}}=\left(\begin{array}{cc}
h_{\mathbf{k}} & \Delta_{\mathbf{k}} \\
\Delta_{\mathbf{k}}^{\dagger} & -h_{-\mathbf{k}}^{\mathrm{T}}
\end{array}\right)
$$

and the four-component Nambu spinor $\Phi_{\mathbf{k}}=$ $\left(c_{\mathbf{k} \uparrow}, c_{\mathbf{k} \downarrow}, c_{-\mathbf{k} \uparrow}^{\dagger}, c_{-\mathbf{k} \downarrow}^{\dagger}\right)^{\mathrm{T}}$, where $c_{\mathbf{k} \sigma}^{\dagger}\left(c_{\mathbf{k} \sigma}\right)$ creates (annihilates) an electron with spin $\sigma$ and momentum $\mathbf{k}$. The normal-state dispersion of the electrons is given by $h_{\mathbf{k}}=\varepsilon_{\mathbf{k}} \sigma_{0}+\lambda \mathbf{l}_{\mathbf{k}} \cdot \boldsymbol{\sigma}$, where $\varepsilon_{\mathbf{k}}=t\left(\cos k_{x}+\cos k_{y}\right)-\mu, \boldsymbol{\sigma}=\left(\sigma_{x}, \sigma_{y}, \sigma_{z}\right)^{\mathrm{T}}$ is the vector of Pauli matrices, $\sigma_{0}$ the $2 \times 2$ identity matrix, and $l_{\mathbf{k}}$ represents the Rashba-type spin-orbit coupling potential with $\mathbf{l}_{\mathbf{k}}=\hat{\mathbf{x}} \sin k_{y}-\hat{\mathbf{y}} \sin k_{x}$. Here, $t$ denotes twice the nearest-neighbor hopping integral, $\mu$ is the chemical potential, and $\lambda$ stands for the spin-orbit coupling strength. Due to the absence of inversion symmetry, the superconducting order parameter $\Delta_{\mathbf{k}}$ contains both even-parity spin-singlet and odd-parity spin-triplet pairing components

$$
\Delta_{\mathbf{k}}=f_{\mathbf{k}}\left(\Delta_{\mathrm{s}} \sigma_{0}+\Delta_{\mathrm{t}} \mathbf{l}_{\mathbf{k}} \cdot \boldsymbol{\sigma}\right)\left(i \sigma_{y}\right)
$$

with $f_{\mathbf{k}}=\sin k_{x} \sin k_{y}$, and where $\Delta_{\mathrm{s}}$ and $\Delta_{\mathrm{t}}$ denote the spin-singlet and spin-triplet pairing amplitudes, respectively. Unless otherwise specified, we set $\left(t, \mu, \lambda, \Delta_{\mathrm{s}}, \Delta_{\mathrm{t}}\right)=$ $(2.0,2.0,1.0,0.0,1.0)$ for our numerical calculations. We have checked that different parameter choices do not qualitatively alter our results, as long as the nodal structure of the superconductor remains the same.

The Rashba-type spin-orbit coupling $\lambda \mathbf{l}_{\mathbf{k}}$ splits the normalstate Fermi surface into two helical Fermi surfaces, given by $\xi_{\mathbf{k}}^{ \pm}=\varepsilon_{\mathbf{k}} \pm \lambda\left|\mathbf{l}_{\mathbf{k}}\right|=0$. As a result of the $d_{x y}$-wave form factor $f_{\mathbf{k}}$, the superconducting order parameter $\Delta_{\mathbf{k}}$ on these two helical Fermi surfaces changes sign, leading to eight nodal points where the gap of the quasiparticle spectrum vanishes [see Fig. [1(a)]. These gap closing-points are located at $\left( \pm k_{0}^{\alpha}, 0\right)$ and $\left(0, \pm k_{0}^{\alpha}\right)$ in the two-dimensional Brillouin zone, where

$$
k_{0}^{\alpha}=\arccos \left[\frac{t(\mu-t)+\alpha \lambda \sqrt{\lambda^{2}+\mu(2 t-\mu)}}{t^{2}+\lambda^{2}}\right],
$$

with $\alpha \in\{+,-\}$. The gapless quasiparticle band structure of these Dirac points is protected by a combination of timereversal and particle-hole symmetry, and their stability is guaranteed by the conservation of a quantized topological invariant [cf. Eq. (4)] ${ }^{[1 \mid 20}$ Particle-hole symmetry $\mathcal{C}=U_{C} \mathcal{K}$ and
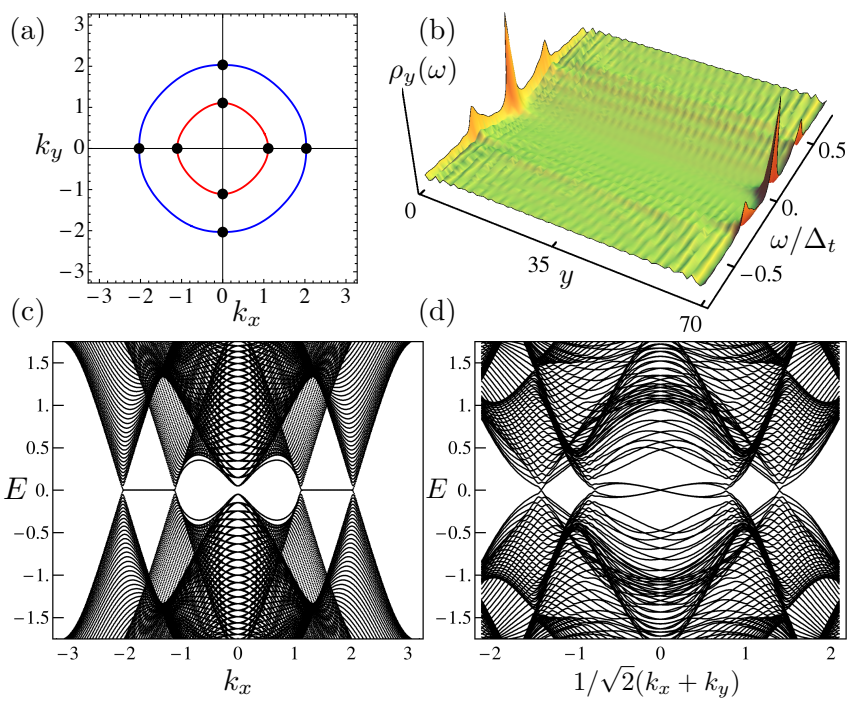

FIG. 1. (Color online) (a) Spin-orbit split Fermi surfaces and nodal points of the superconducting gap $\Delta_{\mathrm{k}}$, Eq. (1). Solid black dots indicate the location of the eight nodal points, where the gap of the quasiparticle spectrum closes. (b) Energy- and layer-resolved density of states $\rho_{y}(\omega)$ of the $\left(d_{x y}+p\right)$-wave superconductor in a ribbon geometry with (01) edge and width $\mathcal{N}_{y}=70$ lattice sites. (c) and (d): Edge band structure of the $\left(d_{x y}+p\right)$-wave superconductor in a ribbon geometry with (01) and (11) edge, respectively, as a function of edge momentum. Zero-energy flat bands appear at the (01) edge connecting the projected nodal points of the two helical Fermi surfaces. These flat-bands give rise to a divergent density of states at $\omega=0$, see panel (b). Note that there are also dispersing edge states, which lead to a feature in the edge density of states at $\omega \simeq \pm 0.3$.

time-reversal symmetry $\mathcal{T}=U_{T} \mathcal{K}$ act on the BdG Hamiltonian (1) as $U_{C} H_{-\mathbf{k}}^{\mathrm{T}} U_{C}^{\dagger}=-H_{\mathbf{k}}$ and $U_{T} H_{-\mathbf{k}}^{\mathrm{T}} U_{T}^{\dagger}=+H_{\mathbf{k}}$, respectively, where $\mathcal{K}$ is the complex conjugation operator, $U_{C}=\sigma_{x} \otimes \sigma_{0}$, and $U_{T}=\sigma_{0} \otimes i \sigma_{y}$. Since $\mathcal{C}^{2}=+1$ and $\mathcal{T}^{2}=-1$, Hamiltonian (1) belongs to class DIII of the symmetry classification. ${ }^{41}$ As a result of these symmetries, $H_{\mathbf{k}}$ anticommutes with the unitary matrix $U_{S}=i \mathcal{T C}=-\sigma_{x} \otimes \sigma_{y}$, i.e., $U_{S}^{\dagger} H_{\mathbf{k}} U_{S}=-H_{\mathbf{k}}$. Hence, $H_{\mathbf{k}}$ takes off-diagonal form in the basis in which $U_{S}$ is diagonal. That is, we have $\widetilde{U}_{S}=W U_{S} W^{\dagger}=\operatorname{diag}\left(\sigma_{0},-\sigma_{0}\right)$, with the transformation matrix

$$
W=\frac{1}{\sqrt{2}}\left(\begin{array}{cc}
\sigma_{0} & -\sigma_{y} \\
\sigma_{0} & \sigma_{y}
\end{array}\right) .
$$

The transformed BdG Hamiltonian reads

$$
\widetilde{H}(\mathbf{k})=W H_{\mathbf{k}} W^{\dagger}=\left(\begin{array}{cc}
0 & D(\mathbf{k}) \\
D^{\dagger}(\mathbf{k}) & 0
\end{array}\right)
$$

where $D(\mathbf{k})=h_{\mathbf{k}} \sigma_{0}+\Delta_{\mathbf{k}} \sigma_{y}$. Due to the chiral symmetry $U_{S}^{\dagger} H_{\mathbf{k}} U_{S}=-H_{\mathbf{k}}$, we can choose the zero-energy eigenfunctions of $H_{\mathbf{k}}$ to be simultaneous eigenstates of $U_{S}$ with a defined chirality eigenvalue $\Gamma=+1$ or $\Gamma=-1$. 16

The topological invariant that guarantees the stability of the eight nodal points of $H_{\mathbf{k}}$ can be defined in terms of the winding number $W_{\mathcal{C}}$ of $\operatorname{det} D(\mathbf{k})$, i.e., in terms of the number 
of revolutions of $\operatorname{det} D(\mathbf{k})$ around the origin of the complex plane as $\mathbf{k}$ moves along a closed contour $\mathcal{C}$. An explicit expression of $W_{\mathcal{C}} \in \mathbb{Z}$ is given by [20, 222]42]

$$
W_{\mathcal{C}}=\frac{1}{2 \pi} \oint_{\mathcal{C}} d k_{l} \partial_{k_{l}}\{\arg [\operatorname{det} D(\mathbf{k})]\},
$$

where $\mathcal{C}$ is a one-dimensional contour encircling one (or several) nodal points in momentum space. As a consequence of the bulk-edge correspondence, a nonzero winding number $W_{\mathcal{C}} \neq 0$ signals the appearance of zero-energy modes at certain edges. For example, for the (01) edge one can define an edge momentum-dependent winding number $W_{\mathcal{C}}\left(k_{x}\right)$ by taking $\mathcal{C}=\left\{\left(k_{x}, k_{y}\right) \mid-\pi \leq k_{y}<\pi\right\}$. For $k_{x}$ between the projected nodes of the two helical Fermi surfaces, $W_{\mathcal{C}}\left(k_{x}\right)$ evaluates to +1 or -1 , which gives rise to a spin-nondegenerate zero-energy flat band at these edge momenta [Fig. 11.c)]. On the (11) edge, however, zero-energy flat bands are absent [Fig. 1]d)] 1511620

In Sec. IV, we will numerically compute the edge modes of the $\left(d_{x y}+p\right)$-wave superconductor for different edges. We will demonstrate that zero-energy flat-band states generically appear at the boundary for arbitrary edge orientations, except for the (11) edge. But before doing so, we first derive in the following section the lowest-order matrix elements for impurity scattering among the flat-band edge states using an explicit expression for the edge-state wave functions.

\section{FLAT-BAND EDGE-STATE WAVE FUNCTION}

To derive the zero-energy edge-state wave functions, let us consider Hamiltonian (1) on the semi-infinite plane $y>0$, with the (01) edge located at $y=0$. The ansatz for the nondegenerate edge-state wave function is taken to be $\Psi_{k_{x}}=$ $\sum_{\alpha, \beta} C_{\beta}^{\alpha} \psi_{\beta}^{\alpha} e^{\kappa_{\beta}^{\alpha} y} e^{i k_{x} x}$, which decays exponentially into the bulk with inverse decay lengths $\operatorname{Re}\left[\kappa_{\beta}^{\alpha}\right]<0$. In the offdiagonal basis, Eq. (3), the wave function $\Psi_{k_{x}}=\left(\chi_{k_{x}}, \eta_{k_{x}}\right)^{\mathrm{T}}$ can be split into a part $\left(\chi_{k_{x}}, 0\right)^{\mathrm{T}}$ with positive chirality $\Gamma=$ +1 and a part $\left(0, \eta_{k_{x}}\right)^{\mathrm{T}}$ with negative chirality $\Gamma=-1$. Since all the eigenstates of $H_{\mathbf{k}}$ can be chosen to have definite chirality, it follows that $\eta_{k_{x}}=0$ whenever $\chi_{k_{x}} \neq 0$ and vice versa. ${ }^{14}[16$ In addition, we observe that for every edge-state wave function

$$
\Psi_{k_{x}}^{+}=\left(\chi_{k_{x}}, 0\right)^{\mathrm{T}}
$$

with edge momentum $k_{x}$ and $\Gamma=+1$ there is a time-reversed partner

$$
\Psi_{k_{x}}^{-}=\left(0, i \sigma_{y} \chi_{-k_{x}}^{*}\right)^{\mathrm{T}}
$$

with edge momentum $-k_{x}$ and $\Gamma=-1$. Using quasiclassical scattering theory, it is shown in Appendix $\mathrm{A}$ that the zeroenergy edge-state wavefunction $\Psi_{k_{x}}^{+}=\left(\chi_{k_{x}}, 0\right)^{\mathrm{T}}$ for $-k_{\mathrm{F}}^{-}<$ $k_{x}<-k_{\mathrm{F}}^{+}$and $\Delta_{\mathrm{t}}>\Delta_{\mathrm{s}}$ can be explicitly written as $\frac{16122}{2}$

$$
\chi_{k_{x}}=\left(\begin{array}{c}
-2 k_{\mathrm{F}}^{+} k_{\perp}^{-} e^{\kappa_{1}^{+} y}+b_{1} e^{\kappa_{2}^{-} y}+b_{1}^{*} e^{\kappa_{1}^{-} y} \\
2 i a_{1} k_{\perp}^{-} e^{\kappa_{1}^{+} y}-a_{2} i e^{\kappa_{1}^{-} y}-a_{2}^{*} i e^{\kappa_{2}^{-} y}
\end{array}\right) e^{i k_{x} x},(5 \mathrm{c})
$$

where $k_{\perp}^{-}=\sqrt{\left(k_{\mathrm{F}}^{-}\right)^{2}-k_{x}^{2}}$ and $k_{\mathrm{F}}^{ \pm}$denote the Fermi momenta of the two helical Fermi surfaces. The coefficients $a_{1}$, $a_{2}$, and $b_{1}$ depend on the edge momentum $k_{x}$ and are defined below Eq. A7] in Appendix A The inverse decay length $\kappa_{1}^{+}$is purely real, whereas $\kappa_{1}^{-}$and $\kappa_{2}^{-}$are complex conjugate partners, see Eq. (A3). An expression similar to Eq. (5) can be derived for the zero-energy edge states on the opposite edge, i.e., for Hamiltonian (1) on $y<0$, which supports zero-energy flat band states with opposite chirality as compared to Eq. (5).

\section{A. Spin polarization of flat-band edge states}

Surface states of noncentrosymmetric superconductors exhibit a helical spin texture, where the spin orientation of the

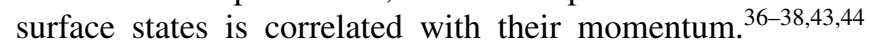
For the (01) edge of the $\left(d_{x y}+p\right)$-wave superconductor one finds that the flat-band states are strongly polarized in the $y z$ spin plane, but have a vanishing spin component along the $x$ axis. ${ }^{36+38}$ Using Eq. (5), it can be explicitly verified that the expectation value of the spin operator

$$
S^{\mu}=\left(\begin{array}{cc}
\sigma^{\mu} & 0 \\
0 & -\left[\sigma^{\mu}\right]^{*}
\end{array}\right), \quad \mu \in\{x, y, z\},
$$

with respect to the surface-state wave functions $\Psi_{k_{x}}^{ \pm}$, Eq. [5], has the following properties (cf. Appendix A

$$
\left\langle\Psi_{k_{x}}^{ \pm}\left|\widetilde{S}^{x}\right| \Psi_{k_{x}}^{ \pm}\right\rangle=0, \quad\left\langle\Psi_{k_{x}}^{ \pm}\left|\widetilde{S}^{y, z}\right| \Psi_{k_{x}}^{ \pm}\right\rangle \neq 0,
$$

for all $k_{x}$ with $k_{\mathrm{F}}^{+}<\left|k_{x}\right|<k_{\mathrm{F}}^{-}$. Here, $\widetilde{S}$ denotes the spin operator in the off-diagonal basis, i.e., $\widetilde{S}^{\mu}=W S^{\mu} W^{\dagger}$. Moreover, one finds that the $y$ component of the spin expectation value is much larger than the $z$ component and that the sign of the $y$-spin polarization correlates with the chirality $\Gamma$ of the flat-band edge state, such that $\operatorname{sgn}\left[\left\langle\Psi_{k_{x}}^{ \pm}\left|\widetilde{S}^{y}\right| \Psi_{k_{x}}^{ \pm}\right\rangle\right]=\mp 1$. Finally, we note that the two flat-band edge states $\Psi_{-k_{x}}^{+}$and $\Psi_{k_{x}}^{-}$, which have opposite edge momenta, have opposite spin polarization. That is,

$$
\left\langle\Psi_{-k_{x}}^{+}\left|\widetilde{S}^{y, z}\right| \Psi_{-k_{x}}^{+}\right\rangle=-\left\langle\Psi_{k_{x}}^{-}\left|\widetilde{S}^{y, z}\right| \Psi_{k_{x}}^{-}\right\rangle,
$$

for all $k_{x}$ with $k_{\mathrm{F}}^{+}<k_{x}<k_{\mathrm{F}}^{-}$, which is consistent with timereversal symmetry.

\section{B. Impurity scattering among flat-band edge states}

In order to calculate the matrix elements for impurity scattering among flat-band edge states, we consider uncorrelated edge disorder described by

$$
H_{\mathrm{imp}}^{\beta}=\sum_{\mathbf{k}, \mathbf{q}} \Phi_{\mathbf{k}}^{\dagger} V_{q_{x}}^{\beta} \Phi_{\mathbf{k}+\hat{\mathbf{e}}_{x} q_{x}},
$$

where $V_{q_{x}}^{\beta}=(1 / \mathcal{N}) \sum_{j} v\left(x_{j}\right) \mathcal{S}^{\beta} e^{-i q_{x} x_{j}}$ denotes the Fourier transform of the impurity potentials $v\left(x_{j}\right) \mathcal{S}^{\beta}$ at the edge sites $x_{j}$ with strengths $v\left(x_{j}\right)$. Here, $\mathcal{N}$ stands for the number of 
lattice sites and $V^{\beta=0}$ corresponds to nonmagnetic impurities with $\mathcal{S}^{0}=\sigma_{z} \otimes \sigma_{0}$, while $V^{\beta=x, y, z}$ represents magnetic exchange scattering with $\mathcal{S}^{x, y, z}=S^{x, y, z}$. First, we observe that impurity scattering processes connecting flat-band edge states to bulk nodal quasiparticles are strongly suppressed, since the bulk density of states vanishes linearly as $\omega \rightarrow 0$ [see Fig. 11(b)]. A rough estimate for the effects of impurity scattering among the zero-energy edges states can be obtained from the matrix elements of the impurity potential $V_{q_{x}}^{\beta}$ between two flat-band edge-state wavefunctions. Because the edge spectrum of the $\left(d_{x y}+p\right)$-wave superconductor has in general two flat bands with opposite chirality [see Fig. 11.c)], it is useful to distinguish between "intraband" scattering between states with the same chirality and "interband" scattering between states with opposite chirality. From

$$
V_{q_{x}}^{0} U_{S}+U_{S} V_{q_{x}}^{0}=0
$$

and

$$
\left\langle\Psi_{k_{x}}^{ \pm}\left|\widetilde{V}_{k_{x}^{\prime}-k_{x}}^{0}\right| \Psi_{k_{x}^{\prime}}^{ \pm}\right\rangle=0, \quad \text { for all } k_{x}, k_{x}^{\prime},
$$

where $\widetilde{V}_{q_{x}}^{0}=W V_{q_{x}}^{0} W^{\dagger}$, it follows that edge flat bands are protected against nonmagnetic intraband scattering by chiral symmetry. In other words, since nonmagnetic onsite disorder preserves the total chirality number of the superconductor, it can remove edge states only in pairs of opposite chirality. Magnetic impurities, on the other hand, break chiral symmetry, i.e., $V_{q_{x}}^{x, y, z} U_{S}+U_{S} V_{q_{x}}^{x, y, z} \neq 0$, and therefore allow for strong intraband scattering.

For the case of impurity scattering between edge flat bands with opposite chirality we find by use of Eqs. (5a) and (5b) that time-reversal invariance forbids nonmagnetic backscattering between the time-reversed partners $\Psi_{k_{x}}^{-}$and $\Psi_{-k_{x}}^{+}$. That is,

$$
\left\langle\Psi_{-k_{x}}^{+}\left|\widetilde{V}_{2 k_{x}}^{0}\right| \Psi_{k_{x}}^{-}\right\rangle=0
$$

for all $k_{x}$ with $k_{\mathrm{F}}^{+}<k_{x}<k_{\mathrm{F}}^{-}$. Moreover, for two flat-band edge states with nearly opposite momenta $k_{x}$ and $-k_{x}^{\prime}$ (i.e., $0<\left|k_{x}-k_{x}^{\prime}\right|<k_{\mathrm{F}}^{-}-k_{\mathrm{F}}^{+}$), one finds that the corresponding matrix element $\left\langle\Psi_{-k_{x}^{\prime}}^{+}\left|\widetilde{V}_{k_{x}+k_{x}^{\prime}}^{0}\right| \Psi_{k_{x}}^{-}\right\rangle$is nonzero but small, due to the mismatch between the almost opposite spin polarizations of the two edge states. In the presence of magnetic impurities, however, spin-flip scattering is allowed, and hence scattering between states with opposite spin polarizations is possible.

The above considerations suggest that moderately strong nonmagnetic disorder, with disorder strength $\gamma_{\text {imp }}$ smaller or of the same order as the superconducting gaps $\left|\Delta_{ \pm}\right|=$ $\left|\Delta_{\mathrm{S}} \pm \Delta_{\mathrm{t}}\right|$, has only weak effects on the flat-band edge states. Magnetic impurities, however, which lift the symmetry protection of the flat-band states, are expected to strongly reduce the number of edge states. To test these expectations, we perform in the following two sections numerical simulations of the $\left(d_{x y}+p\right)$-wave superconductor in the presence of different types of edge disorder.

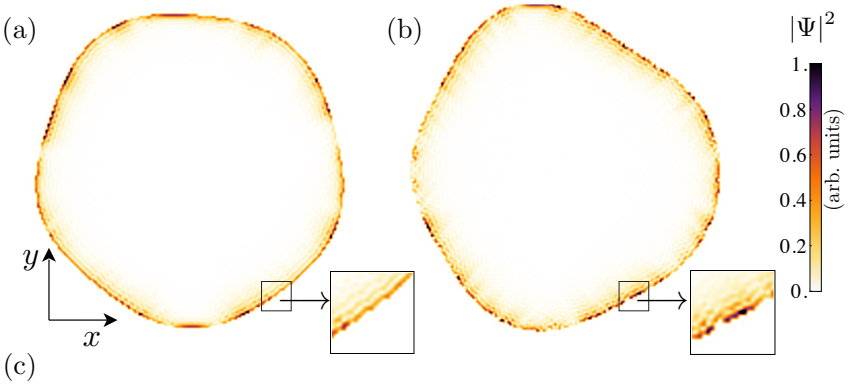

(c)

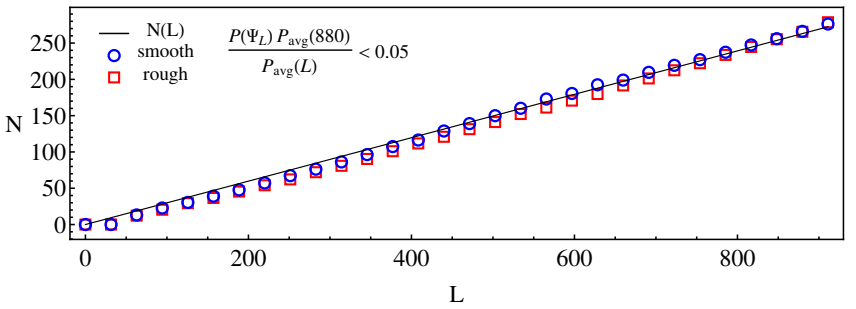

FIG. 2. (Color online) Density plot of the edge-state wave function amplitudes $\left|\Psi_{L}\right|^{2}$ in a $\left(d_{x y}+p\right)$-wave superconducting dot with (a) smooth and (b) rough boundaries. Edge states are present both at an irregular but smooth boundary (a) and at a boundary with short-range disorder (b). Panel (c) shows the average number of edge states for an ensemble of randomly shaped superconducting dots with smooth (blue circles) and rough (red squares) boundaries as a function of circumference $L$ of the dot ${ }^{[4]}$ Here, the edge states are separated from the bulk states according to criterion 15 and by additionally requiring that the energy of the states is smaller than $0.1\left|\Delta_{ \pm}\right|$in absolute value. The solid black line represents the analytical approximation given by Eq. (17).

\section{EDGE STATES AT IRREGULARLY SHAPED BOUNDARIES}

In order to compute the edge-state wave functions of an irregularly shaped $\left(d_{x y}+p\right)$-wave superconduting dot with smooth or rough edges, we Fourier transform Hamiltonian (1) to real space and diagonalize it using standard eigenvalue algorithms ${ }^{[46}$ The shape of the superconducting dot is defined in terms of a direction-dependent radius ${ }^{47}$

$$
R(\theta)=\sum_{i=1}^{5} w_{i} \sin \left(i \theta-\phi_{i}\right)
$$

with the parameters $w_{i}$ and $\phi_{i}$ and the angle of direction $\theta$. With this definition, the dots can be constructed by cutting the shapes given by Eq. (13) out of a square lattice grid. This results in superconducting dots with smooth edges, whose orientation is locally well defined [Fig. 2/a)]. Bulk- and edgestate wave functions can be distinguished in terms of the participation ratio $P\left(\Psi_{L}\right)$ of a given eigenstate $\Psi_{L}\left(\mathbf{r}_{i}\right)$ of a dot with circumference $L$, i.e., $\frac{48}{48}$

$$
P\left(\Psi_{L}\right)=\frac{\left(\sum_{i}\left|\Psi_{L}\left(\mathbf{r}_{i}\right)\right|^{2}\right)^{2}}{\mathcal{N} \sum_{i}\left|\Psi_{L}\left(\mathbf{r}_{i}\right)\right|^{4}},
$$

where $i$ runs over all the sites $\mathbf{r}_{i}$ in the $\operatorname{dot}$ and $\mathcal{N}$ is the total number of sites. The participation ratio $P\left(\Psi_{L}\right)$ represents 
the number of lattice sites occupied by the Bogoliubov quasiparticle wave function $\Psi_{L}$ compared to the total number of sites $\mathcal{N}$. Hence, for extended bulk states $P\left(\Psi_{L}\right) \simeq 1$, whereas for localized edge states $P\left(\Psi_{L}\right) \ll 1$. We find that for sufficiently large dots, a good characterization of the edge-state wave functions is given by

$$
P\left(\Psi_{L}\right) \frac{P_{\mathrm{avg}}(880)}{P_{\mathrm{avg}}(L)}<0.05,
$$

where $P_{\text {avg }}(L)=\frac{1}{n} \sum_{\iota=1}^{n} \frac{1}{\left|\mathcal{W}_{\iota}\right|} \sum_{\Psi_{L}^{\iota} \in \mathcal{W}_{\iota}} P\left(\Psi_{L}^{\iota}\right)$ is the average participation ratio of all the low-energy wave functions $\Psi_{L}^{\iota}$ of an ensemble of randomly shaped superconducting dots of circumference $L$. Here, $n$ denotes the size of the statistical ensemble and $\mathcal{W}_{\iota}$ is the set of the first $\sim L / 2$ lowest positive energy wave functions calculated numerically for each sample. ${ }^{46}$ Since edge disorder leads to a small $L$-dependent decrease of the participation ratio $P\left(\Psi_{L}\right)$ of all the wave functions $\Psi_{L}$, we have included in Eq. (15) the renormalization factor $P_{\text {avg }}(880) / P_{\text {avg }}(L)$, where $L=880$ is the circumference of the largest dots.

a. Smooth edges. We first study irregularly shaped dots with smooth boundaries, where the edge orientation is locally well defined [inset of Fig. 2(a)]. These boundaries consist of both (01)-edge and (11)-edge type parts, leading to long-range correlated disorder. As exemplified in Fig. 22a), we find that ingap states appear at almost all boundaries of the dot. That is, the behavior characteristic of the (01)-edge [Fig. 1. (1)] is generic and qualitatively independent of the edge orientation. Hence, the number of edge states is expected to scale linearly with the circumference $L$ of the superconducting dot. Within a simplified continuum theory, one can show that the density of edge states per unit length for a smooth edge is approximately given by (cf. Appendix A

$$
\frac{d N}{d l}=\left|k_{0}^{-} \sin \varphi-k_{0}^{+} \cos \varphi\right|-\left|k_{0}^{-} \cos \varphi-k_{0}^{+} \sin \varphi\right|,
$$

where $0 \leq \varphi<\pi / 4$ is the angle between the local edge orientation and the nearest (01) or (10) direction. Integrating Eq. (16) along the circumference, we find that the total number of edge states for a circular dot is given by

$$
\begin{aligned}
N(L) & =\int_{0}^{L} \frac{d N}{d l} d l \\
& =\frac{8 L}{\pi}\left[\frac{k_{0}^{-}+k_{0}^{+}}{\sqrt{2}}+\frac{k_{0}^{-}-k_{0}^{+}}{2}-\sqrt{\left(k_{0}^{-}\right)^{2}+\left(k_{0}^{+}\right)^{2}}\right] .
\end{aligned}
$$

As it turns out, Eq. (17) is a good approximation for the number of edge states of an irregularly shaped dot. This is revealed in Fig. 2(c), which shows the average number of edge states as a function of $L$ for an ensemble of randomly shaped superconducting dots with smooth boundaries (blue circles $4 \sqrt{45}$ together with the analytical result, Eq. (17). The numerical data and the analytical curve are in good agreement except for dots with small circumferences, with $L<50$, where finitesize effects become important.

b. Rough edges. Second, we consider rough boundaries with edge disorder on the lattice scale. In order to introduce short-range edge disorder, we start from the smooth edges,
Eq. (13), and randomly extract edge sites with probability $p_{\mathrm{rm}}=0.01$, while moving around the edge of the dot once ${ }^{47}$ This "etching" process is repeated twenty times, which leads to an irregular boundary with both long-range and short-range correlated disorder [inset of Fig. 2[b)]. The edge-state wave function amplitudes for a superconducting dot with rough edges is plotted in Fig. 2(b). As in the case of smooth edges, we find that edge states appear at almost all boundaries. That is, short-range edge disorder does not change the total number of edge states, but only shifts some of the edge states away from zero energy. This is further evidenced in Fig 2 (c) (red squares), which shows that the average number of ingap states at a randomly shaped boundary with short-range disorder scales linearly in $L$, and is in good agreement with Eq. (17).

In conclusion, our numerical simulations of $\left(d_{x y}+p\right)$-wave superconducting dots with short-range and long-range edge disorder demonstrate that the bulk-edge correspondence remains valid even in the absence of translation symmetry. Ingap states generically appear at the boundary of these superconductors, for almost all edge orientations. Due to their topological origin (cf. Sec. IIIB), the edge states are robust against nonmagnetic scattering from both short-range and long-range correlated edge disorder.

\section{STRONG EDGE DISORDER}

Let us now investigate in detail the effects of strong edge disorder, which affects both edge and bulk states, leading to a nontrivial interaction between the two. In order to access larger system sizes than in Sec. IV] we employ here recursive Green's function techniques ${ }^{49 \sqrt{50}}$ to calculate the lattice Green's function $G(\omega ; \mathbf{r})$ of a disordered $\left(d_{x y}+p\right)$-wave superconducting ribbon (see Appendix B). From the Green's function $G(\omega ; \mathbf{r})$ the local density of states in the $y$-th layer is obtained via

$$
\rho_{y}(\omega)=-\frac{1}{4 \pi} \frac{1}{\mathcal{N}_{x}} \sum_{x} \operatorname{Im}[\operatorname{Tr}\{G(\omega ; x, y)\}],
$$

where $\mathcal{N}_{x}$ denotes the length of the superconducting ribbon. In the following, we have considered samples of width $\mathcal{N}_{y}=70$ sites and length $\mathcal{N}_{x}=600$ sites. Quenched edge disorder is implemented by adding random on-site potentials $V_{x_{j}}^{\beta}=v\left(x_{j}\right) \mathcal{S}^{\beta}$ in the two outermost layers of the superconducting ribbon. We consider two different types of

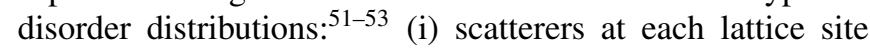
with local potentials $v\left(x_{j}\right)$ drawn from a box distribution $p\left[v\left(x_{j}\right)\right]=1 / \gamma_{\text {imp }}$ for $v\left(x_{j}\right) \in\left[-\gamma_{\text {imp }} / 2,+\gamma_{\text {imp }} / 2\right]$ (referred to as "Gaussian" type disorder),, and (ii) a dilute density $\rho_{\text {imp }}$ of strong scatterers with constant potential strength $v\left(x_{j}\right) \equiv v_{\text {imp }} \gtrsim\left|\Delta_{ \pm}\right|$(referred to as "unitary" type disorder). In case (i) the strength of the disorder is controlled by the width $\gamma_{\text {imp }}$ of the distribution, whereas in case (ii) it can be adjusted in terms of both the impurity density $\rho_{\text {imp }}$ and the potential strength $v_{\text {imp }}$. Since fluctuations between different disorder realizations are minor, we present in Figs. 3 and 4 

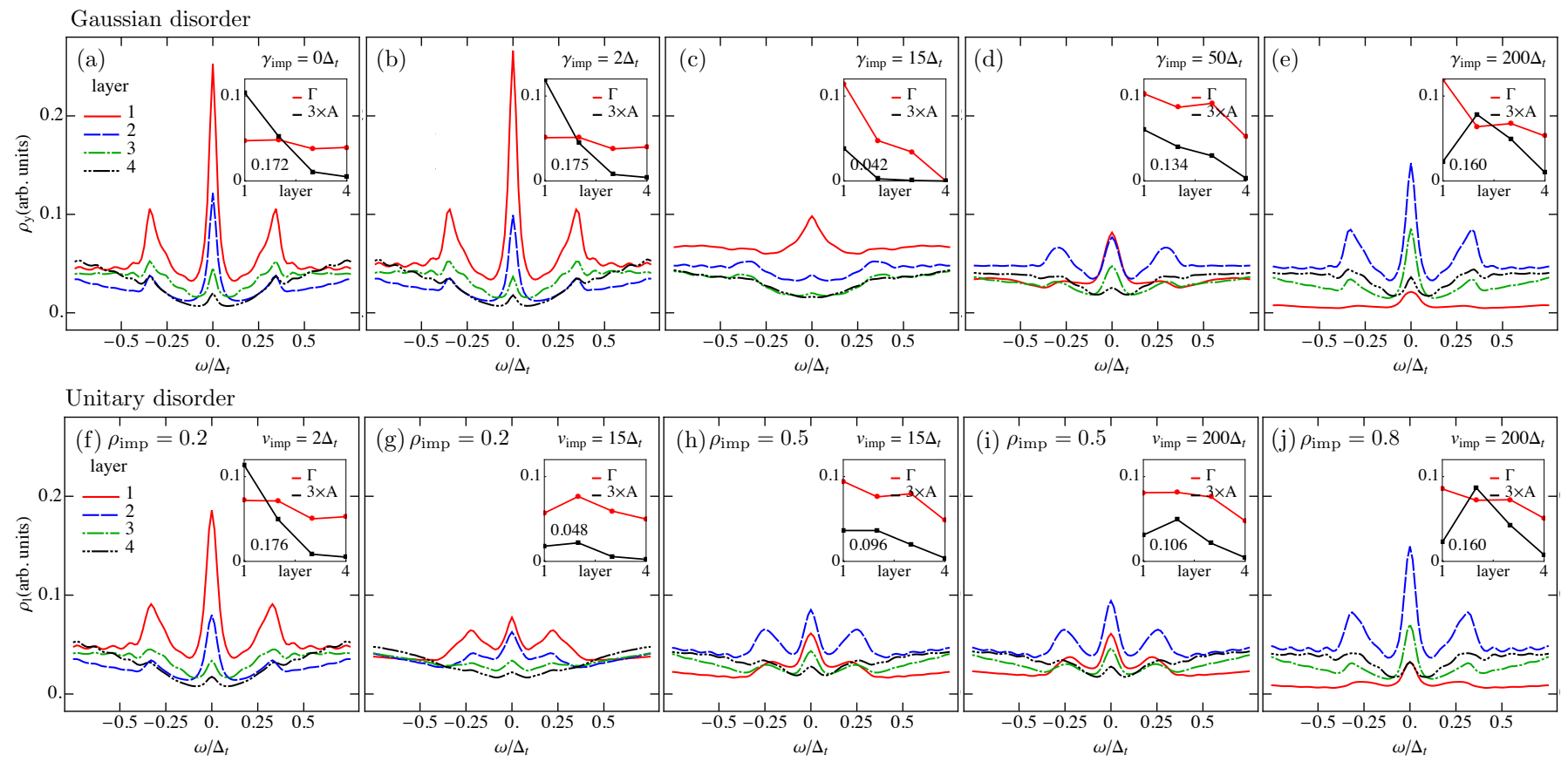

FIG. 3. (Color online) Layer-resolved local density of states $\rho_{y}(\omega)$ plotted for the first four outermost layers of a $\left(d_{x y}+p\right)$-wave superconducting ribbon with (01) edges in the presence of (a)-(e) "Gaussian" edge disorder and (f)-(j) "unitary" edge disorder (for details see text). The insets show the width $\Gamma$ and the area $A$ of the Lorentzian peaks at $\omega=0$ as a function of layer index $y$. The number in the lower left corner of the insets indicates the total area of the zero-bias peak as obtained by summing $A$ over the first four layers. In the clean case, $v_{\text {imp }}=0$, the edge states penetrate only about two layers into the bulk [panel (a)]. For strong disorder the outermost layer shows signatures of localization, while new weakly disordered states appear in the second and third inward layers [panels (e) and (j)].

spectra for a specific disorder realization, without averaging over disorder configurations.

\section{A. Nonmagnetic impurities}

We start by discussing the effects of nonmagnetic impurities with potentials $V_{x_{j}}^{0}=v\left(x_{j}\right) \mathcal{S}^{0}$. In Fig. 3 is shown the local density of states $\rho_{y}(\omega)$ for the first four outermost layers of a $\left(d_{x y}+p\right)$-wave superconducting ribbon with nonmagnetic disorder of different strengths. The case of "Gaussian" type disorder is plotted in panels (a)-(e), whereas the effects of "unitary" type disorder are presented in panels (f)-(j). In order to estimate the number of ingap edge states in the system, we have fitted a Lorentzian function to the zero-bias peaks in Fig. 3. The peak width $\Gamma$ and the peak area $A$ provide a measure for the number of edge states and their spread in energy, respectively (insets in Fig. 3). In agreement with the analytical arguments given in Sec. IIIB, we find that weak and even moderately strong disorder, with $\gamma_{\text {imp }}$ (or $\rho_{\text {imp }} v_{\text {imp }}$ ) of the same order as the superconducting gaps $\left|\Delta_{ \pm}\right|$, has very little effect on the edge states: Gaussian disorder gives rise to a slightly faster decay of the edge states into the bulk [Fig. 3. b)], whereas unitary disorder somewhat increases the energy spread of the ingap states [Fig. 3(f)]. The total number of edge states, however, is unaffected by moderately strong disorder [compare insets in Figs. 3(a), 3. b), and 3 ff)].
For strong edge disorder with $\gamma_{\text {imp }} \gg\left|\Delta_{ \pm}\right|$(or $\rho_{\text {imp }} v_{\text {imp }} \gg$ $\left|\Delta_{ \pm}\right|$), on the other hand, the states in the outermost layer become strongly localized. But remarkably, new weakly disordered edge states appear at the second and third inward layers [Fig. 3 (e) and $3(\mathrm{j})]$. In other words, due to the bulk-boundary correspondence, zero-energy states emerge at the interface between the bulk topological superconductor and the Anderson insulator formed by the outermost layer. This behavior is reminiscent of topological-insulator surface states perturbed by strong disorder $[5455$

\section{B. Magnetic impurities}

Magnetic impurities $V_{x_{j}}^{x, y, z}=v\left(x_{j}\right) \mathcal{S}^{x, y, z}$ break timereversal symmetry, thereby lifting the symmetry protection of the edge states. In Fig. 4 we present the edge density of states $\rho_{\text {edge }}$, defined as the sum of $\rho_{y}(\omega)$ over the four outermost layers, of a $\left(d_{x y}+p\right)$-wave superconducting ribbon with (01) edges in the presence of impurity spins polarized along the $x, y$, and $z$ axes [panels (b)-(d) and (g)-(i)] and randomly oriented magnetic disorder [panels (e) and (j)]. For comparison, Figs. 4(a) and 4(f) show the edge density of states for nonmagnetic scalar impurities. As before, we consider both "Gaussian" type disorder [Figs. 4(a)-(e)] and "unitary" type disorder [Figs. [4(f)-(j)]. Since the flat-band edge states are polarized within the $y z$ spin-plane (cf. Sec. [III), impurity spins polarized along the $y$ and $z$ axes couple strongly 

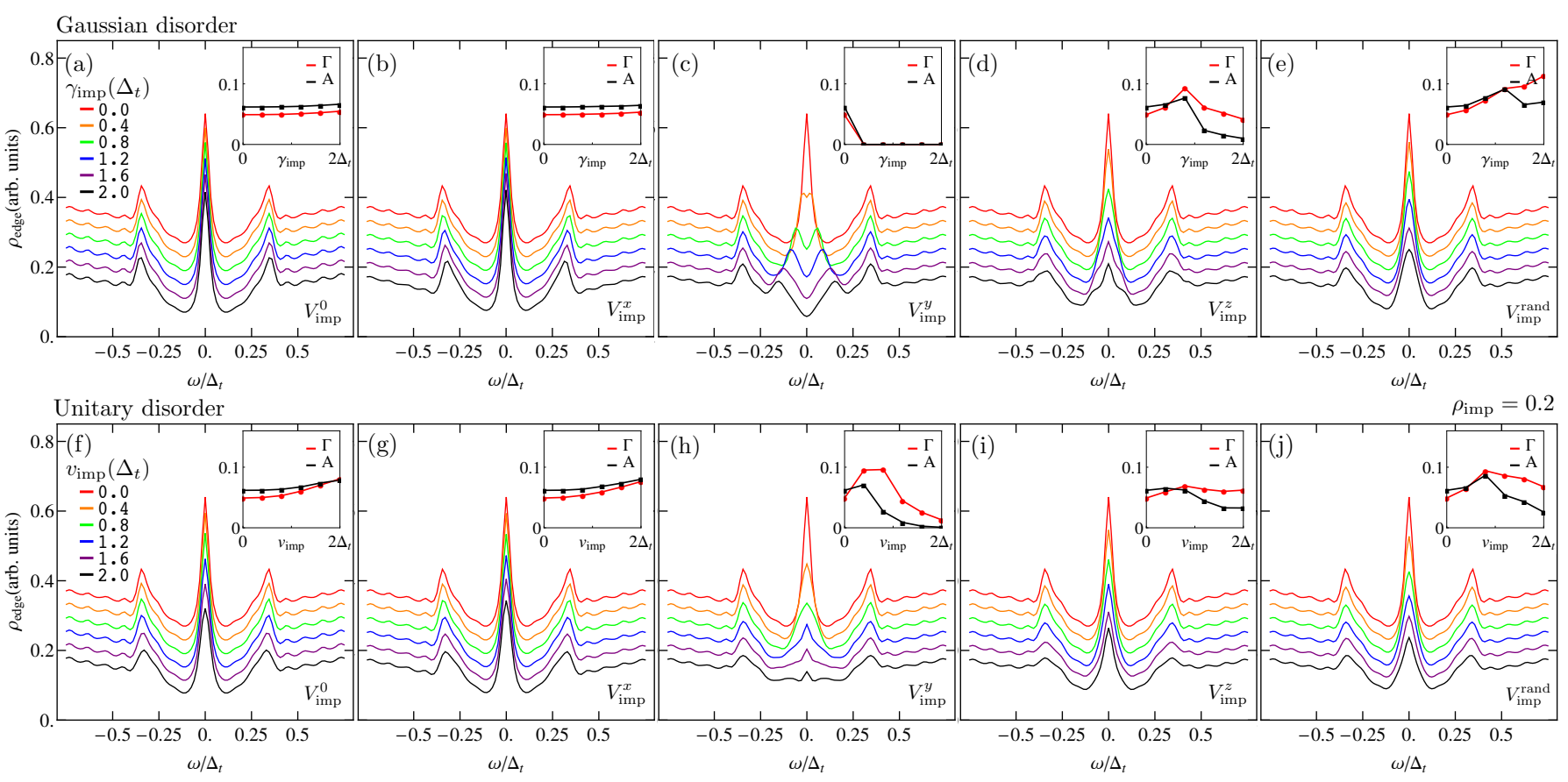

FIG. 4. (Color online) Local density of states summed over the four outermost layers, $\rho_{\text {edge }}(\omega)=\frac{1}{4} \sum_{y=1}^{4} \rho_{y}(\omega)$, of a $\left(d_{x y}+p\right)$-wave superconducting ribbon with (01) edges in the presence of nonmagnetic [panels (a) and (f)] and magnetic impurities [panels (b)-(e) and (g)-(j)] in the two outermost layers. Two different disorder distributions are considered: (a)-(e) "Gaussian" disorder and (f)-(j) "unitary" disorder with $\rho_{\text {imp }}=0.2$ (for details see text). Individual traces are vertically offset by 0.02 from one another for clarity. The insets show the width $\Gamma$ and the area $A$ of the Lorentzian peaks at $\omega=0$ as a function of disorder strengths $\gamma_{\text {imp }}$ and $v_{\text {imp }}$, respectively.

to the flat bands, whereas scalar impurities and $x$ spin polarized impurities leave the edge states almost unaffected as long as $\gamma_{\text {imp }}\left(\rho_{\text {imp }} v_{\text {imp }}\right)$ is not much larger than $\left|\Delta_{ \pm}\right|$. As shown in Figs. $4(\mathrm{c})$ and 4 (h), $y$ spin polarized impurities are particularly harmful to the flat-band edge states, even for relatively small disorder strengths of $\gamma_{\text {imp }} \simeq 0.8\left|\Delta_{ \pm}\right|$(or $v_{\text {imp }} \simeq 0.8\left|\Delta_{ \pm}\right|$for the "unitary" type disorder).

\section{SUMMARY AND CONCLUSIONS}

In summary, we have shown that flat-band edge states in noncentrosymmetric superconductors are robust against weak and moderately strong nonmagnetic edge disorder, as long as the disorder strength is not much larger than the superconducting gaps. Using analytical considerations, we have found that spin-independent scattering among the flat-band edge states is suppressed due to the definite chirality of the edge-state wave functions and their helical spin texture (Sec. III B). By means of extensive numerical simulations, we have demonstrated that moderately strong spin-independent disorder spreads the zero-energy edge states over a small band in energy, but does not alter the total number of edge states [Figs. 4(a) and 4(f)]. However, in the presence of strong edge disorder, with disorder strength much larger than the superconducting gaps, the wave functions in the outermost layer localize, but new weakly disordered ingap states appear in the second and third inward layers [Figs. 3(e) and 3(j)]. We have investigated the edge orientation dependence of the edge state density by numerically simulating superconducting dots with both smooth and rough boundaries. Edge states appear for almost all edge orientations, even in the absence of translation symmetry along the boundary [Fig. 2]. This demonstrates that translation symmetry is not crucial for the protection of the edge states. Time-reversal and particle-hole symmetry, on the other hand, play a key role for the stability of the flat-band states. Consequently, we have found that magnetic impurities, which break time-reversal symmetry, substantially decrease the number of edge states even for small impurity densities [Figs. 4(c) and 4(h)].

Nondegenerate flat-band edge states are expected to appear in any nodal topological superconductor with strong Rashba type spin-orbit coupling, such as, e.g., $\mathrm{CePt}_{3} \mathrm{Si}$ or $\mathrm{Li}_{2} \mathrm{Pt}_{3} \mathrm{~B}$. These boundary states can in principle be observed using scanning tunneling microscopy or angle-resolved photoemission spectroscopy. The signature of the flat-band edge states on transport in various heterostructures involving topological superconductors remains as a direction for future research, as well as the study of interaction effects among the flat-band edge states $\left[\frac{56}{6}\right.$

\section{ACKNOWLEDGMENTS}

The authors thank P. Brydon, C.-K. Chiu, A. Damascelli, J. Hofmann, P. Ostrovsky, S. Ryu, C. Timm, and P. Wahl for 
useful discussions.

\section{Appendix A: Derivation of zero-energy edge-state wave function}

In order to derive Eq. (5), we perform a small momentum expansion of tight-binding Hamiltonian (1), around the $\Gamma$-point. This yields a continuum model with quadratic dispersions in the normal state and Fermi wave vectors

$$
k_{\mathrm{F}}^{\alpha}=-\alpha m \lambda+\sqrt{(m \lambda)^{2}+2 m \widetilde{\mu}},
$$

where $m=-1 / t, \widetilde{\mu}=\mu-2 t$, and $\alpha \in\{+,-\}$ labels the two helical Fermi surfaces. As in the main text, we consider a (01) edge located at $y=0$, where the superconductor and the vacuum occupy the half-spaces $y>0$ and $y<0$, respectively. The zero-energy edge states can be determined by solving the equation $H\left(k_{x},-i \partial_{y}\right) \Psi_{k_{x}}=0$, with the wave function ansatz $\Psi_{k_{x}}=\Psi_{k_{x}} e^{\kappa y}$. Here, $\operatorname{Re}[\kappa]$ is the inverse decay length of the edge state. In the following, we focus on solutions with positive chirality $\Gamma=+1$, which exist within the interval $-k_{\mathrm{F}}^{+}<k_{x}<-k_{\mathrm{F}}^{-}$. In that case the secular equation, $\operatorname{det}\left[H\left(k_{x},-i \kappa\right)\right]=0$, can be reexpressed as

$$
\begin{aligned}
\operatorname{det}[ & \left.D^{\dagger}\left(k_{x},-i \kappa\right)\right]=\left[\kappa k_{x} \Delta_{\mathrm{s}}+\tilde{\mu}-\frac{t}{2}\left(\kappa^{2}-k_{x}^{2}\right)\right]^{2} \\
& +\left(\lambda-\kappa k_{x} \Delta_{\mathrm{t}}\right)^{2}\left(\kappa^{2}-k_{x}^{2}\right)=0,
\end{aligned}
$$

which is a polynomial equation of fourth degree in $\kappa$. The nature of the roots of Eq. A2 can be inferred, to some extent, from the free term $a_{0}=t^{2}\left(k_{x}^{2}-k_{\mathrm{F}}^{-2}\right)\left(k_{x}^{2}-k_{\mathrm{F}}^{+2}\right) / 4$ of this quartic equation. An explicit expression for the roots of Eq. (A2) can be given within the quasi-classical approximation. For $-k_{\mathrm{F}}^{+}<k_{x}<-k_{\mathrm{F}}^{-}$, we have $a_{0}<0$, and Eq. A2 for $\Delta_{\mathrm{t}}>\Delta_{\mathrm{s}}$ has two real roots and two complex conjugate roots. That is, the solutions of Eq. $\mathrm{A} 2$ ) are given by

$$
\begin{aligned}
& \kappa_{\beta}^{+}=-k_{\perp}^{+}-(-1)^{\beta} i \frac{k_{\mathrm{F}}^{+}}{k_{\perp}^{+}} \sqrt{\frac{\widetilde{\Delta}_{+}^{2}\left[k_{x}, i k_{\perp}^{+}\right]}{\lambda^{2}+2 \widetilde{\mu} / m}}, \\
& \kappa_{\beta}^{-}=(-1)^{\beta+1} i k_{\perp}^{-}-\frac{k_{\mathrm{F}}^{-}}{k_{\perp}^{-}} \sqrt{\frac{\widetilde{\Delta}_{-}^{2}\left[k_{x}, k_{\perp}^{-}\right]}{\lambda^{2}+2 \widetilde{\mu} / m}},
\end{aligned}
$$

with $\beta \in\{1,2\}$, the transverse momenta $k_{\perp}^{+}=\sqrt{k_{x}^{2}-\left(k_{\mathrm{F}}^{+}\right)^{2}}$ and $k_{\perp}^{-}=\sqrt{\left(k_{\mathrm{F}}^{-}\right)^{2}-k_{x}^{2}}$, and $\widetilde{\Delta}_{ \pm}[\mathbf{k}]=\left(k_{\mathrm{F}}^{ \pm} \Delta_{\mathrm{t}} \pm \Delta_{\mathrm{s}}\right) k_{x} k_{y}$. We observe that $\kappa_{1}^{+}$and $\kappa_{2}^{+}$are purely real, while $\kappa_{1}^{-}$and $\kappa_{2}^{-}$ form a complex conjugate pair. Furthermore, the maximum decay length $\max \left\{-\operatorname{Re}\left[\kappa_{\beta}^{\alpha}\right]^{-1}\right\}$ rapidly increases as $k_{x} \rightarrow$ $-k_{\mathrm{F}}^{ \pm}$. In other words, the wave functions are well confined to the edge for $k_{x}$ in the middle of the interval $\left[-k_{\mathrm{F}}^{+},-k_{\mathrm{F}}^{-}\right.$, whereas as $k_{x}$ approaches the boundaries of the interval the flat-band states start to penetrate over longer distances into the bulk.

For each of the four roots $\kappa_{\beta}^{ \pm}$, the kernel of the secular equation is spanned by one basis vector $\psi_{\beta}^{ \pm}$, which reads in the off-diagonal basis, Eq. (3),

$$
\psi_{\beta}^{+}=\left(2-\beta, \frac{i(2-\beta) k_{\mathrm{F}}^{+}}{k_{\perp}^{+}-k_{x}}, \beta-1, \frac{i(\beta-1) k_{\mathrm{F}^{+}}}{k_{\perp}^{+}-k_{x}}\right)^{\mathrm{T}},
$$

$$
\psi_{\beta}^{-}=\left(1, \frac{-k_{\mathrm{F}}^{-}}{i k_{x}+(-1)^{\beta} k_{\perp}^{-}}, 0,0\right)^{\mathrm{T}},
$$

with $\beta \in\{1,2\}$. With this, the ansatz for the flat-band edge states can be written as a linear combination of the basis states $\mathrm{A} 4$ )

$$
\Psi_{k_{x}}=\sum_{\alpha \in\{+,-\}} \sum_{\beta \in\{1,2\}} C_{\beta}^{\alpha} \psi_{\beta}^{\alpha} e^{\kappa_{\beta}^{\alpha} y} e^{i k_{x} x}
$$

where the coefficients $C_{\beta}^{\alpha}$ are fixed by the boundary conditions

$$
\Psi\left(k_{x}, y=0\right)=0, \quad \Psi\left(k_{x}, y=\infty\right)=0 .
$$

The latter condition implies $C_{2}^{+}=0$, since $\operatorname{Re}\left[\kappa_{2}^{+}\right]>0$, but there exists a nonzero solution for $\left(C_{1}^{+}, C_{1}^{-}, C_{2}^{-}\right)$that satisfies the boundary conditions. After some algebra, we find that in the off-diagonal basis, Eq. (3), the zero-energy edgestate wave function with positive chirality $\Gamma=+1$ is given by $\Psi_{k_{x}}^{+}=\left(\chi_{k_{x}}, 0\right)^{\mathrm{T}}$, with

$$
\chi_{k_{x}}=\left(\begin{array}{c}
-2 k_{\mathrm{F}}^{+} k_{\perp}^{-} e^{\kappa_{1}^{+} y}+b_{1} e^{\kappa_{2}^{-} y}+b_{1}^{*} e^{\kappa_{1}^{-} y} \\
2 i a_{1} k_{\perp}^{-} e^{\kappa_{1}^{+} y}-a_{2} i e^{\kappa_{1}^{-} y}-a_{2}^{*} i e^{\kappa_{2}^{-} y}
\end{array}\right) e^{i k_{x} x},
$$

where $a_{1}=k_{\perp}^{+}+k_{x}, a_{2}=a_{1}\left(k_{\perp}^{-}+i k_{x}\right)+i k_{\mathrm{F}}^{+} k_{\mathrm{F}}^{-}$, and $b_{1}=k_{\mathrm{F}}^{+}\left(k_{\perp}^{-}+i k_{x}\right)+i k_{\mathrm{F}}^{-} a_{1}$.

Similarly, we can derive solutions of the equation $H\left(k_{x},-i \partial_{y}\right) \Psi_{k_{x}}=0$ with negative chirality $\Gamma=-1$, which exist within the interval $k_{\mathrm{F}}^{+}<k_{x}<k_{\mathrm{F}}^{-}$. Repeating similar steps as above, we find that the negative chirality edge-state wave function is given by $\Psi_{k_{x}}^{-}=\left(0, \eta_{k_{x}}\right)^{\mathrm{T}}$, with

$$
\eta_{k_{x}}=\left(\begin{array}{c}
-2 i \widetilde{a}_{1} k_{\perp}^{-} e^{\kappa_{1}^{+} y}+\widetilde{a}_{2} i e^{\kappa_{1}^{-} y}+\widetilde{a}_{2}^{*} i e^{\kappa_{2}^{-} y} \\
2 k_{\mathrm{F}}^{+} k_{\perp}^{-} e^{\kappa_{1}^{+} y}-\widetilde{b}_{1}^{*} e^{\kappa_{1}^{-} y}-\widetilde{b}_{1} e^{\kappa_{2}^{-} y}
\end{array}\right) e^{i k_{x} x},
$$

where $\widetilde{a}_{1}=k_{\perp}^{+}-k_{x}, \widetilde{a}_{2}=\widetilde{a}_{1}\left(k_{\perp}^{-}-i k_{x}\right)+i k_{\mathrm{F}}^{-} k_{\mathrm{F}}^{+}$, and $\widetilde{b}_{1}=k_{\mathrm{F}}^{+}\left(k_{\perp}^{-}-i k_{x}\right)+i k_{\mathrm{F}}^{-} \widetilde{a}_{1}$. As expected, the wave functions $\Psi_{k_{x}}^{+}=\left(\chi_{k_{x}}, 0\right)$ and $\Psi_{k_{x}}^{-}=\left(0, \eta_{k_{x}}\right)$ transform into each other by time-reversal symmetry, i.e.,

$$
\left(\begin{array}{cc}
0 & i \sigma_{y} \\
i \sigma_{y} & 0
\end{array}\right) \Psi_{k_{x}}^{+}(y)=\left[\Psi_{-k_{x}}^{-}(y)\right]^{*},
$$

where we have used the fact that $\kappa_{1}^{+}$is purely real and $\left(\kappa_{1}^{-}, \kappa_{2}^{-}\right)$are complex conjugate partners.

Using Eqs. (A7) and (A8), the expectation value of the spin operator $\widetilde{S}^{\mu}$ can be computed in a straightforward manner. In particular, we find that $\left\langle\Psi_{k_{x}}^{+}\left|\widetilde{S}^{x}\right| \Psi_{k_{x}}^{+}\right\rangle=0$, since the first component of $\chi_{k_{x}}$, Eq. (A7], is purely real, whereas the second component of $\chi_{k_{x}}$ is purely imaginary. Likewise, we have $\left\langle\Psi_{k_{x}}^{-}\left|\widetilde{S}^{x}\right| \Psi_{k_{x}}^{-}\right\rangle=0$.

In closing, we remark that for other edge orientations the flat-band states can be derived in a similar manner as above. For any given edge orientation we can define the momentum parallel to the edge as $k_{\|}=k_{x} \sin (\varphi)+k_{y} \cos (\varphi)$. Correspondingly, the secular equation for the positive chirality 


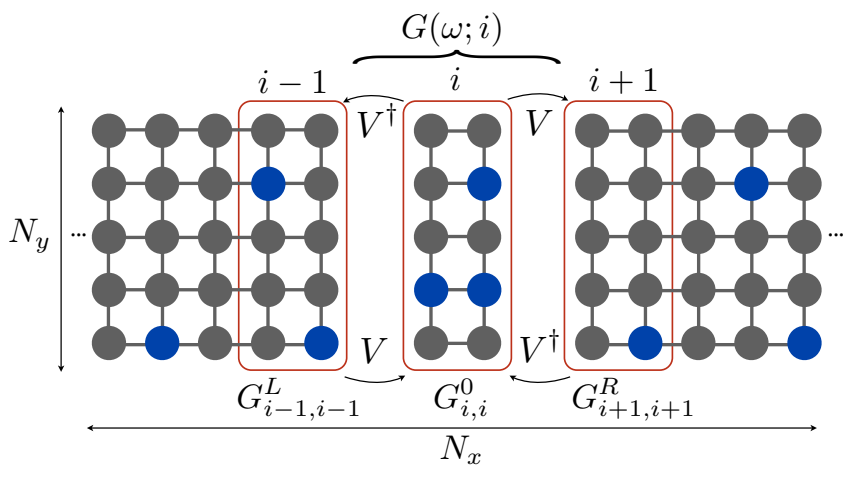

FIG. 5. (Color online) Scheme of the recursive Green's function method. To accommodate second neighbor hopping an effective building block of two columns is considered, as well as an effective coupling $V$. The local Green's function $G(\omega ; i)$ is calculated by attaching to the block $i$ the left $\left(G^{L}\right)$ and right $\left(G^{R}\right)$ ribbons according to Eq. B7). The blue sites represent random on-site disorder potentials, defined by $H_{\mathrm{imp}}^{\beta}(x)$.

edge states reads $\operatorname{det}\left[D^{\dagger}\left(k_{\|},-i \kappa\right)\right]=0$. Within the continuum approximation, we find that for dominant triplet pairing, $\Delta_{\mathrm{t}}>\Delta_{\mathrm{s}}$, the flat bands are always of single degeneracy $!^{17 / 22}$ Zero-energy states appear in regions of the edge Brillouin zone that are bounded by the projected nodal points. Hence, the density of edge states per unit length can be approximated by

$\frac{d N}{d l}=\left|k_{0}^{-} \sin \varphi-k_{0}^{+} \cos \varphi\right|-\left|k_{0}^{-} \cos \varphi-k_{0}^{+} \sin \varphi\right|,(\mathrm{A} \mid$

where $\varphi \in[0, \pi / 4[$ is the angle between the considered edge orientation and the nearest (01) or (10) direction.

\section{Appendix B: Recursive Green's function technique}

The local density of states of a disordered superconductor can be efficiently computed using the recursive Green's function technique ${ }^{4950}$ This is achieved by considering a discrete ribbon in real space with width of $\mathcal{N}_{y}$ sites and length of $\mathcal{N}_{x}$ sites. Let us define the block Hamiltonian $H^{n}$ corresponding to the coupling of two columns spaced by $n$ lattice sites in the $x$ direction,

$$
H^{n}=\frac{1}{(2 \pi)^{2}} \int d^{2} \mathbf{k} H_{\mathbf{k}} e^{i k_{y}\left(y-y^{\prime}\right)} e^{i n k_{x}},
$$

where $H^{0}$ corresponds to a free column Hamiltonian, while $H^{1}$ and $H^{2}$ are the coupling to the nearest and next nearest neighbouring columns, respectively. We have considered in our simulations on-site impurities as defined in Eq. (9). For each ribbon's column, i.e., fixed $x$ in $\mathbf{r}=(x, y)$, we have

$$
H_{\mathrm{imp}}^{\beta}(x)=\sum_{i=1, N_{\mathrm{imp}}} \Phi_{\mathbf{r}}^{\dagger} v_{\mathrm{imp}}\left(\mathbf{r}_{\mathbf{i}}\right) \delta_{\mathbf{r}, \mathbf{r}_{i}} S^{\beta} \Phi_{\mathbf{r}},
$$

with impurity positions, $\mathbf{r}_{\mathbf{i}}$, defined according to the disorder distribution, see Sec. V] To accommodate next-nearest neighbour hopping in a convenient way, we redefine the building blocks of the ribbon to be $8 \mathcal{N}_{y} \times 8 \mathcal{N}_{y}$-matrices incorporating two columns as the building blocks, by writing

$$
\begin{aligned}
H_{i}^{\beta} & =\left(\begin{array}{cc}
H^{0}+H_{\mathrm{imp}}^{\beta}(2 i-1) & H^{1} \\
H^{1 \dagger} & H^{0}+H_{\mathrm{imp}}^{\beta}(2 i)
\end{array}\right), \\
V & =\left(\begin{array}{cc}
H^{2} & 0 \\
H^{1} & H^{2}
\end{array}\right) .
\end{aligned}
$$

Here, the block index $i$ runs from 1 to $\mathcal{N}_{x} / 2$, i.e., with spacing of 2 lattice sites. Dyson's equation for $G(\omega ; i) \equiv G_{i, i}$ takes the form

$$
G_{i, i}=G_{i, i}^{0}+G_{i, i}^{0} V G_{i-1, i}^{L}+G_{i, i}^{0} V^{\dagger} G_{i, i+1}^{R},
$$

where the $\omega$ dependence is suppressed for simplicity, $G_{i, i}^{0}=$ $\left[\omega+i \eta-H_{i}^{\beta}\right]^{-1}$ stands for the uncoupled block at $i$, while $G_{i, j}^{L / R}$ represent the ribbons to its left and right,

$$
G_{i-1, i}^{L}=G_{i-1, i-1}^{L} V G_{i, i}, \quad G_{i, i+1}^{R}=G_{i+1, i+1}^{R} V^{\dagger} G_{i, i} .
$$

The side ribbons are calculated recursively, using the relations

$$
\begin{aligned}
& G_{n, n}^{L(n)}=\left[\omega+i \eta-H_{n-1}^{\beta}-V^{\dagger} G_{n-1, n-1}^{L(n-1)} V\right]^{-1}, \\
& G_{n, n}^{R(n)}=\left[\omega+i \eta-H_{n+1}^{\beta}-V G_{n+1, n+1}^{R(n+1)} V^{\dagger}\right]^{-1},
\end{aligned}
$$

at iteration step $n$, where $n \in\{1, \ldots, i-1\}$ and $n \in$ $\left\{\mathcal{N}_{x} / 2, \ldots, i+1\right\}$ for $G^{L}$ and $G^{R}$, respectively. Finally, the local Green's function can be reduced to

$$
G_{i, i}=\left[\omega+i \eta-H_{i}^{\beta}-\Sigma_{i}^{L}-\Sigma_{i}^{R}\right]^{-1},
$$

with self-energies, $\Sigma_{i}^{L / R}$, given by

$$
\Sigma_{i}^{L}=V^{\dagger} G_{i-1, i-1}^{L} V, \quad \Sigma_{i}^{R}=V G_{i+1, i+1}^{R} V^{\dagger} .
$$

The local density of states can then be easily computed by Eq. (18), where $G(\omega ; x, y)$ are obtained from the diagonal entries of $G(\omega ; i)$.

\footnotetext{
* r.queiroz@ fkf.mpg.de

a.schnyder@fkf.mpg.de

1 A. P. Schnyder, S. Ryu, A. Furusaki, and A. W. W. Ludwig, Phys.
}

Rev. B 78, 195125 (2008).

2 A. Kitaev, AIP Conference Proceedings 1134, 22 (2009).

3 M. Z. Hasan and C. L. Kane, Rev. Mod. Phys. 82, 3045 (2010). 
4 S. Ryu, A. P. Schnyder, A. Furusaki, and A. W. W. Ludwig, New Journal of Physics 12, 065010 (2010).

5 A. P. Schnyder, P. M. R. Brydon, D. Manske, and C. Timm, Phys. Rev. B 82, 184508 (2010).

6 X.-L. Qi and S.-C. Zhang, Rev. Mod. Phys. 83, 1057 (2011).

7 C. Beenakker, Annual Review of Condensed Matter Physics 4, 113 (2013).

8 J. Alicea, Reports on Progress in Physics 75, 076501 (2012).

9 S. Sasaki, M. Kriener, K. Segawa, K. Yada, Y. Tanaka, M. Sato, and Y. Ando, Phys. Rev. Lett. 107, 217001 (2011).

10 V. Mourik, K. Zuo, S. M. Frolov, S. R. Plissard, E. P. A. M. Bakkers, and L. P. Kouwenhoven, 336, 1003 (2012).

11 A. Das, Y. Ronen, Y. Most, Y. Oreg, M. Heiblum, and H. Shtrikman, Nat Phys 8, 887 (2012).

${ }^{12}$ M. T. Deng, C. L. Yu, G. Y. Huang, M. Larsson, P. Caroff, and H. Q. Xu, Nano Letters 12, 6414 (2012).

${ }^{13}$ M. Mondal, B. Joshi, S. Kumar, A. Kamlapure, S. C. Ganguli, A. Thamizhavel, S. S. Mandal, S. Ramakrishnan, and P. Raychaudhuri, Phys. Rev. B 86, 094520 (2012).

14 A. P. Schnyder and S. Ryu, Phys. Rev. B 84, 060504 (2011).

15 Y. Tanaka, Y. Mizuno, T. Yokoyama, K. Yada, and M. Sato, Phys. Rev. Lett. 105, 097002 (2010).

${ }^{16}$ M. Sato, Y. Tanaka, K. Yada, and T. Yokoyama, Phys. Rev. B 83, 224511 (2011)

17 P. M. R. Brydon, A. P. Schnyder, and C. Timm, Phys. Rev. B 84, 020501 (2011).

18 J. P. Dahlhaus, M. Gibertini, and C. W. J. Beenakker, Phys. Rev. B 86, 174520 (2012).

19 C. L. M. Wong, J. Liu, K. T. Law, and P. A. Lee, Phys. Rev. B 88, 060504 (2013).

${ }^{20}$ K. Yada, M. Sato, Y. Tanaka, and T. Yokoyama, Phys. Rev. B 83, 064505 (2011).

${ }^{21}$ B. Beri, Phys. Rev. B 81, 134515 (2010).

22 A. P. Schnyder, P. M. R. Brydon, and C. Timm, Phys. Rev. B 85, 024522 (2012).

${ }^{23}$ S. Matsuura, P.-Y. Chang, A. P. Schnyder, and S. Ryu, New Journal of Physics 15, 065001 (2013).

${ }^{24}$ E. Bauer and M. Sigrist, Non-Centrosymmetric Superconductors: Introduction and Overview, vol. 847 of Lecture Notes in Physics (Springer Berlin, 2012).

25 S. Fujimoto, Journal of the Physical Society of Japan 76, 051008 (2007).

${ }^{26}$ E. Bauer, G. Hilscher, H. Michor, C. Paul, E. W. Scheidt, A. Gribanov, Y. Seropegin, H. Noël, M. Sigrist, and P. Rogl, Phys. Rev. Lett. 92, 027003 (2004).

${ }^{27}$ K. Izawa, Y. Kasahara, Y. Matsuda, K. Behnia, T. Yasuda, R. Settai, and Y. Onuki, Phys. Rev. Lett. 94, 197002 (2005).

${ }^{28}$ I. Bonalde, R. L. Ribeiro, W. Brmer-Escamilla, C. Rojas, E. Bauer, A. Prokofiev, Y. Haga, T. Yasuda, and Yanuki, New Journal of Physics 11, 055054 (2009).

${ }^{29}$ I. Sugitani, Y. Okuda, H. Shishido, T. Yamada, A. Thamizhavel, E. Yamamoto, T. D. Matsuda, Y. Haga, T. Takeuchi, R. Settai, et al., J. Phys. Soc. Jpn. 75, 043703 (2006).

${ }^{30}$ H. Mukuda, T. Fujii, T. Ohara, A. Harada, M. Yashima, Y. Kitaoka, Y. Okuda, R. Settai, and Y. Onuki, Phys. Rev. Lett. 100,
107003 (2008).

${ }^{31}$ H. Q. Yuan, D. F. Agterberg, N. Hayashi, P. Badica, D. Vandervelde, K. Togano, M. Sigrist, and M. B. Salamon, Phys. Rev. Lett. 97, 017006 (2006).

${ }^{32}$ M. Nishiyama, Y. Inada, and G.-q. Zheng, Phys. Rev. Lett. 98, 047002 (2007).

33 P. Badica, T. Kondo, and K. Togano, Journal of the Physical Society of Japan 74, 1014 (2005).

${ }^{34}$ G. Eguchi, D. C. Peets, M. Kriener, S. Yonezawa, G. Bao, S. Harada, Y. Inada, G.-q. Zheng, and Y. Maeno, Phys. Rev. B 87, 161203 (2013).

35 Y. Tanaka, T. Yokoyama, A. V. Balatsky, and N. Nagaosa, Phys. Rev. B 79, 060505 (2009).

${ }^{36}$ P. M. R. Brydon, C. Timm, and A. P. Schnyder, New Journal of Physics 15, 045019 (2013).

37 A. P. Schnyder, C. Timm, and P. M. R. Brydon, Phys. Rev. Lett. 111, 077001 (2013).

38 J. S. Hofmann, R. Queiroz, and A. P. Schnyder, Phys. Rev. B 88, 134505 (2013).

${ }^{39}$ G. M. Graf and M. Porta, ArXiv e-prints (2012), 1207.5989.

40 Note that for fully gapped systems there exist real-space formulas for the topological invariants, see, e.g., Refs. 57 and 58

41 A. Altland and M. R. Zirnbauer, Phys. Rev. B 55, 1142 (1997).

${ }^{42}$ Note that, while Hamiltonian $H_{\mathbf{k}}$ defined on the entire twodimensional Brillouin zone belongs to symmetry class DIII, $H_{\mathbf{k}}$ restricted to a one-dimensional noncentrosymemtric contour in the Brillouin zone belongs to class AIII.

43 A. B. Vorontsov, I. Vekhter, and M. Eschrig, Phys. Rev. Lett. 101, 127003 (2008)

44 C.-K. Lu and S. Yip, Phys. Rev. B 82, 104501 (2010).

${ }^{45}$ For each member of the ensemble of irregularly shaped dots the weights $w_{i}$ and phases $\phi_{i}$ in Eq. (13) are randomly chosen from the intervals ] $0,0.05[$ and $] 0,2 \pi[$, respectively.

${ }^{46}$ R. B. Lehoucq, D. C. Sorensen, and C. Yang, ARPACK Users' Guide: Solution of Large-Scale Eigenvalue Problems with Implicitly Restarted Arnoldi Methods, Society for Industrial and Applied Mathematics (1997).

${ }^{47}$ M. Wimmer, A. R. Akhmerov, and F. Guinea, Phys. Rev. B 82, 045409 (2010).

${ }^{48}$ R. J. Bell and P. Dean, Discuss. Faraday Soc. 50, 55 (1970).

49 A. C. Potter and P. A. Lee, Phys. Rev. B 83, 094525 (2011).

${ }^{50}$ P. Lee and D. Fisher, Phys. Rev. Lett. 47, 882 (1981).

51 A. P. Schnyder, Ph.D. thesis, ETH Zürich (2007).

52 W. A. Atkinson, P. J. Hirschfeld, A. H. MacDonald, and K. Ziegler, Phys. Rev. Lett. 85, 3926 (2000).

53 C. Chamon and C. Mudry, Phys. Rev. B 63, 100503 (2001).

${ }^{54}$ G. Schubert, H. Fehske, L. Fritz, and M. Vojta, Phys. Rev. B 85, 201105 (2012).

55 Z. Ringel, Y. E. Kraus, and A. Stern, Phys. Rev. B 86, 045102 (2012).

56 Y. Li, D. Wang, and C. Wu, New Journal of Physics 15, 085002 (2013).

57 I. Mondragon-Shem, J. Song, T. L. Hughes, and E. Prodan, ArXiv e-prints (2013), 1311.5233.

${ }^{58}$ M. B. Hastings and T. A. Loring, Journal of Mathematical Physics 51, 015214 (2010). 